\title{
Transplantation of bone mesenchymal stem cells effectively promotes intestine regeneration to eliminate endotoxemia and reduce mortality in the animal model of acute liver failure
}

\author{
Ting Jiang \\ Kunming Medical University \\ Geng Xia \\ Kunming Medical University \\ Bo Yang \\ Kunming Medical University \\ Hong-wei Zhang
}

Chinese Academy of Medical Sciences and Peking Union Medical College

Yue-shan Yin

Kunming Medical University

Cheng-wei Tang

Sicuan University

Jin-hui Yang ( $\sim 17787003686 @ 163 . c o m$ )

The Second Affiliated Hospital of Kunming Medical University https://orcid.org/0000-0002-7234-0923

\section{Research}

Keywords: Bone marrow mesenchymal stem cells, acute liver failure, endotoxemia, differentiation, coculture

Posted Date: May 15th, 2020

DOl: https://doi.org/10.21203/rs.3.rs-27569/v1

License: (9) This work is licensed under a Creative Commons Attribution 4.0 International License. Read Full License 


\section{Abstract \\ Background}

Endotoxemia based on liver failure has been reported to be related to the worse clinical outcomes, but its management remains unsatisfactory. The addition of bone marrow mesenchymal stem cells (BMSCs) could promote the recovery of liver function and increase the survival with the liver failure. However, little is known about the potential of cell therapy with endotoxemia based on liver failure.

\section{Methods}

BMSCs were isolated from rats, and their morphology, differentiation potential, surface markers, and cell cycle were assayed. Thioacetamide-induced acute liver failure rats were randomized to groups with or without BMSCs. During the experiment, survival was recorded. Diamine oxidase (DAO), endotoxin, interleukin-6 (IL-6) and tumor necrosis factor- alpha (TNF-a) and tissue were analyzed by enzyme-linked immunosorbent assay (ELISA), histology, and western blot. Bromodeoxynucleoside uracil (BrdU) incorporation assay was performed to observe the migration of BMSCs. The intestinal epithelial differentiation of BMSCs was induced by co culture with small intestinal crypt in rats (IEC-6). Immunofluorescence was used to analyze the expression of intestinal endothelial markers. Western blot analysis was further performed to examine the differentiation effect when inhibiting the phosphoinositide kinase-3 (PI3K)/protein kinase B (AKT)/mammalian target of rapamycin (mTOR) signaling pathway.

\section{Results}

BrdU-traced BMSCs targeted migrate to intestinal injury sites. Mortality was significantly decreased and intestinal damage was repaired following BMSCs transplantation. Proteomics revealed higher expression of DAO, endotoxin, IL- 6 and TNF- $a$ in the model animals, but these changes were reversed after BMSCs transplantation. In the in vitro study, the intestinal epithelial differentiation of BMSCs was exhibited following co-culture. Moreover, the blocking of PI3K/AKT/mTOR signal pathway inhibited this differentiation.

\section{Conclusions}

These evidences indicate that BMSCs eliminate endotoxemia and reduce mortality in the animal model of acute liver failure by reducing intestine damage.

\section{Background}

Acute liver failure is characterized by sudden development of liver dysfunction and rapid hepatocellular necrosis, may progress to exhibit hepatic coma even death due to various of etiologies, including drug 
toxicity, hepatic ischemia, immune-mediated attack, and viral hepatitis such as hepatitis B and hepatitis C $[1,2]$. Endotoxemia represents the severe stage of acute liver failure and is associated with poor quality of life, mortality, and economic burden [3]. Reasons for severe endotoxemia mainly include intestinal and hepatic factors, which also interact with each other [4]. Although liver transplantation is considered as a curative treatment, the disparity between demand and supply and its long time and heavy price limit the applicability $[5,6]$. As the exhibition of endotoxemia is very urgent, hepatic coma and death are caught soon. Therefore, at the same time of saving hepatocytes, prevention and intervention of the endotoxemia are particularly important.

Endotoxin are mainly produced by overgrowth of gram-negative bacteria in damaged gut with the increased permeability. The increased damage of intestine in the liver failure is usually associated with liver dysfunction, congestion of portal vein, and bacterial infection [7]. The degradation of many harmful protein metabolites such as putrescine, histamine, spermine, and spermidine is catalyzed in the liver and obstructed when hepatocellular necrosis occurs. The high level of toxic substances is not only harmful to brain cells called hepatic coma, but also a great challenge to enterocytes. Meanwhile, the increased portal vein pressure caused by liver failure leads to intestinal congestion, which increases the accumulation of harmful substances in the intestine and affects the intestinal immune responses, cell proliferation, and apoptosis. Moreover, the bacterial infection has been demonstrated to increase the damage of intestine and causes bacteremia or even sepsis. Thus, a huge burden for intestine through internal and external negative factors not only endangers the intestine itself, but also aggravates the condition of the liver, forming malignant circle [8]. However, the effect of current treatment for endotoxemia in liver failure is not satisfactory.

Mesenchymal stem cells (MSCs) immediately attracted a lot of interests owing to the excellent ability of self-renewal and differentiation into various tissues. Furthermore, compared with embryonic (ESCs) and induced pluripotent stem cells (iPSCs), MSCs are easily isolated from bone marrow called the bone marrow mesenchymal stem cells (BMSCs) and without the limitation of ethical problems, the way of cell retention and immunological rejection $[9,10]$. Recently, studies have described the applications of BMSCs for liver diseases. BMSCs could migrate to the injured liver and regenerate in the mice receiving partial hepatectomy $(\mathrm{PH})$ and even improve the alleviation of liver fibrosis $[11,12]$ Moreover, the application of BMSCs have demonstrated the potential for the alleviation of liver injury and inflammation by inhibiting inflammatory factors' activation [13]. However, few researches focus on the role of intestine and the core is made no mention of the gut when it involves stem cells in the liver failure.

In this study, we aimed to assess whether the administration of BMSCs could have beneficial effects both in gut and liver in an animal model with acute liver failure. Moreover, we explored the possibly signalingbased mechanism underlying this therapy.

\section{Methods And Materials}


The study obeyed the laboratory animal welfare and ethic principle, and all the experimental animal procedures were carried out strictly in accordance with the protocol approved by Animal Experimental Ethical Committee of Kunming Medical University (Approval number KMMU2018020).

\section{Isolation and culture of rat BMSCs}

The BMSCs were isolated from SD rats (half male and female, 6-8 weeks old, $200 \pm 10 \mathrm{~g}$ ) which purchased from the experimental animal center of Kunming Medical University (Number of qualification: SCXK20150002). BMSCs were maintained in clean cell culture bottles in maintenance medium containing highglucose Dulbecco's modified Eagle's medium (DMEM; Hyclone, Logan, Utah, USA), 15\% fetal bovine serum (FBS; Hyclone), $100 \mathrm{U} / \mathrm{ml}$ penicillin (Hyclone), and $100 \mu \mathrm{g} / \mathrm{ml}$ streptomycin (Hyclone). In order to observe the morphology and obtain more cells for following studies, cells were placed in an incubator at $37^{\circ} \mathrm{C}$ and $5 \% \mathrm{CO}^{2}$ for $3-4$ passages.

\section{In vitro adipogenic differentiation and oil red 0 staining}

The cells in passage 3 were cultured with adipogenic medium (Saiye Bio, Guangzhou, China) for 16 days in order to induce adipogenic differentiation. After 18 days, cells were stained with Oil Red O (Saiye Bio). In brief, cells were washed with phosphate buffer saline (PBS; Hyclone) and fixed with $4 \%$ paraformaldehyde (Saiye Bio) for 30 minutes. After washing with PBS (Hyclone) three times, cells were incubated with Oil Red O working solution for 30 minutes. Haematoxylin (Saiye Bio) was used for intensifying the staining of the nuclei. Red colour lipid droplets and purple nucleus could be checked microscopically in the final step.

\section{Flow cytometry}

Cells harvested from SD rats were treated with trypsin (Hyclone) in the third generation, washed twice with PBS (Hyclone), and incubated with anti-CD34 (Santa Cruz Biotechnology, sc-7324, USA), anti-CD 45 (Biolegend, 202207, San Diego, USA), anti-CD90 (Biolegend, 202526) and anti-CD29 antibodies (Abcam, ab36219, Cambridge, UK) at room temperature in dark for half an hour. A FACS flow cytometer was used for Analysis.

\section{Cell-Cycle Analysis}

For cell-cycle analysis, cells were stained with propiony iodide (Pl; Biyuntian Bio, Shanghai, China) and analyzed using a flow cytometer.

\section{An animal model of acute liver failure and cell transplantation}

In this study, acute liver failure was induced by thioacetamide (TAA; Sigma-Aldrich, Bornem, Belgium) as previously described [14-16]. To get an ideal animal model, we divided the rats into groups to explore the best TAA dose and check point. The rats were randomly allocated to 4 groups ( $n=25$ each). Group 1 (control group) received saline, and group 2-4 (model groups) received different doses (200mg, 300mg, 
$400 \mathrm{mg} / \mathrm{Kg} /$ day) of TAA via gavage. Then, after confirming the appropriate dose of modeling, we transplanted BMSCs to rats with liver failure, rats were divided into 3 groups: saline, TAA + saline, and TAA + saline + BMSCs $\left(1 \times 10^{6}\right.$, intravenous injection). To collect samples, all rats were sacrificed by $\mathrm{CO}^{2}$ asphyxiation.

\section{Histology}

Samples (livers and intestines) were fixed in $4 \%$ paraformaldehyde (Saiye Bio), dehydrated, and embedded in paraffin. Sections $(4 \mu \mathrm{m})$ were stained with hematoxylin and eosin (H\&E; Saiye Bio). Images ( $\times 100$ magnification) were taken from each sample, and histopathological evaluation of the entire section was performed in a blinded manner. The injury of liver and intestine was scored using the modified histopathologic score described previously [17-19]. These criteria for histological score were shown in Table 1.

\section{Bromodeoxynucleoside uracil (BrdU) Assay}

BrdU incorporation assay was performed to observe the location of BMSCs. Cells were treated with BrdU for 60 min following the manufacturer's instructions (FITC BrdU Flow Kit, CST, Shanghai, China) before the administration.

\section{Determination of diamine oxidase (DAO) and endotoxin}

DAO activity was used a kit (Sigma). In brief, $3 \mathrm{~mL}$ of phosphate buffer $(0.2 \mathrm{~mol} / \mathrm{L}, \mathrm{pH} 7.2), 0.1 \mathrm{~mL}(4 \mu \mathrm{g})$ of horseradish peroxidase solution, $0.1 \mathrm{~mL}$ of $o$-dianisidine methanol solution, $0.5 \mathrm{~mL}$ of plasma and 0.1 $\mathrm{mL}$ of substrate solution ( $175 \mu \mathrm{g}$ of cadaverine dihydrochloride) made up the assay mixture. The DAO activity could be measured as the absorbance at $436 \mathrm{~nm}$ when incubated for 30 minutes at $37^{\circ} \mathrm{C}$. The endotoxin of plasma was assayed by the test kit according to the manufacturer's instructions (Pierce ${ }^{\text {TM }}$ Chromogenic Endotoxin Quant Kit, Thermo, Shanghai, China).

\section{Enzyme-linked immunosorbent assay (ELISA)}

The levels of interleukin-6 (IL-6) and tumor necrosis factor- alpha (TNF-a) in serum were determined according to the manufacturer's instruction of the ELISA kit (IL-6, BMS625, Thermo; TNF-a, KRC3011, Thermo). The content was calculated according to the standard curve and OD value. Data statistics were carried out for the average value of the test results.

\section{Immunofluorescence analysis}

Cells were fixed with PBS (Hyclone) containing 4\% paraformaldehyde (Saiye Bio) for $30 \mathrm{~min}$ at room temperature, and blocked for another $30 \mathrm{~min}$. The cells were then incubated with primary antibodies (Cytokeratin (CK), ab68260, Abcam; Villin, ab201989, Abcam) overnight at $4^{\circ} \mathrm{C}$ and further incubated with secondary antibody (FITC conjugated IgG; Merck Millipore, USA). Olympus phase-contrast fluorescent 
microscope was used for capturing the images. Scoring was based on the previously proposed method [20].

\section{Transwell co-culture system}

Transwell chambers (Millipore) were used in the co-culture induction system. Briefly, the lower chamber was loaded with BMSCs $\left(1 \times 10^{6}\right.$ cells/well) and the upper insert was loaded with epithelial cells of small intestinal crypt in rats (IEC-6; $1 \times 10^{6}$ cells/well) which were obtained from Kunming Cell Bank of Chinese Academy of Sciences (No: KCB200720YJ). The induction system was cultured in an incubator with $5 \%$ $\mathrm{CO}^{2}$ at $37^{\circ} \mathrm{C}$ for 10 days. to interfere with the phosphoinositide kinase-3 (PI3K)/protein kinase $\mathrm{B}$ (AKT)/mammalian target of rapamycin (mTOR) signaling pathway, the inhibitor LY294002 (Abcam, ab120243) was added to the co-culture system.

\section{Western blot analysis}

Cells and intestines were homogenized and lysed following $30 \mathrm{~min}$ incubation on ice. The lysates were centrifuged at 12,000 rpm for $10 \mathrm{~min}$. Proteins isolated from samples were resolved on $10 \%$ sodium dodecyl sulfate-polyacrylamide gel electrophoresis (SDS-PAGE) gels and then were transferred to a polyvinylidene fluoride (PVDF) membrane (Merck Millipore). Blots were blocked for $1 \mathrm{~h}$ at room temperature with $5 \%$ nonfat dried milk. After washing in tris-buffered saline Tween-20 (TBST, Saiye Bio), the membranes were immunoblotted with the following antibodies: anti-IL-6 (Abcam, ab9770), anti-TNF-a (Abcam, ab66579), anti-AKT (Abcam, ab8805), anti-mTOR (Abcam, ab32028), anti-CK (Abcam, ab115293), anti-Villin (Abcam, ab109516), anti- $\beta$ actin (Abcam, ab8227). The blots were then incubated with the corresponding horseradish peroxidase-conjugated IgG (Abcam, ab7090, ab7125). Mylmage (SLB, Korea) was used to detect the immunoreactive proteins, and Labworks 4.0 software was used for quantitative analysis (UVP Inc., USA).

\section{Statistical analysis}

The GraphPad Prism version 7 program (GraphPad Software, Inc., San Diego, CA, USA) was used for graphs. Data are presented as mean \pm standard deviation. Differences between multiple groups were analyzed by one-way analysis of variance (ANOVA) or two-way ANOVA. A P value of $<0.05$ was considered statistically significant.

\section{Results}

Identification of BMSCs from rats was revealed by their typical morphology and potency to differentiate to multilineage as well as their immunophenotypic analysis as showed later.

The bone marrow of SD rats was used for isolation of the BMSCs. These cells showed a fibroblast-like, spindle-shaped morphology at passage PO (Fig. 1a) and gradually adherence to the bottom of culture bottle without differences between groups (Fig. 1b). The BMSCs must be differentiated to various cell 
types to demonstrate their pluripotency. Then we used the adipogenic differentiation medium to check the potency. Oil Red $\mathrm{O}$ staining revealed droplets of fat in the adipogenic induction group of BMSCs (Fig. $1 \mathrm{c}$ and Fig. 1d). The cell surface antigens for the BMSCs characterization were detected by flow cytometry. Obviously, we could notice that CD90 and CD29 but were negative for CD45 and CD34 (Fig. 1g and Fig. 1h). Also, in order to ensure the follow-up experiments, we tested the cell cycle of these separated BMSCs. As shown in Fig. 1e and Fig. 1f, these cells were in a phase of proliferation to meet experimental requirements

\section{Effects of different doses of TAA on animal model}

Obvious pathological manifestations of intestine injury based on acute liver failure and appropriate survival rate were two basis this study would be performed. Then, we divided the rats into several groups to explore the effect of different doses of TAA (Fig. 2a). Both group of TAA300 $\mathrm{mg} / \mathrm{Kg} / \mathrm{day}$ and group of TAA400 mg $/ \mathrm{Kg} /$ day showed typical hepatoenteropathology of acute liver failure (Fig. 2c and Fig. 2d), while group of TAA400mg/Kg/day had a higher mortality (Fig. 2b), which was difficult to guarantee the follow-up experiments. Therefore, for this study, the dosage of TAA300 $\mathrm{mg} / \mathrm{Kg} / \mathrm{day}$ was adopted.

\section{The administration of BMSCs reduced the mortality of TAA-induced acute liver failure rat and decreased the damage of intestine}

The damage of intestinal mucosal barrier is critical to the progress of liver failure. This damage could induce severe endotoxemia leading to death. To determine whether the administration of BMSCs can solve this clinical problem, we divided the model rats into several groups to observe the effects of BMSCs on survival and pathology (Fig. 3a). Our data showed that the mortality with BMSCs was significantly lower as compared to those without BMSCs (Fig. 3b). The damage of intestine in the group with BMSCs administration was also significantly decreased (Fig. 3c). Moreover, we found out that BrdU-traced BMSCs targeted migrated to intestinal injury sites (Fig. 3d).

\section{BMSCs ameliorated endotoxemia and inflammation of TAA-induced rat}

To characterize the effects of BMSCs on endotoxemia in liver failure rats, DAO and endotoxin in plasma were tested with ELISA. For IL-6 and TNF-a, important inflammatory factors with wide biological effects, were assayed both in plasma and intestine. As shown in Fig. 3e, DAO and endotoxin were significantly less content than the group without BMSCs. This suggests that BMSCs ameliorated endotoxemia, possibly being the result of BMSCs migration and following intestine repair. Furthermore, the inflammatory factors in group with BMSCs, including the IL- 6 and TNF-a, were quantified at a lower level compared to group without BMSCs (Fig. 3e and Fig. 3f).

\section{Association of PI3K/AKT/mTOR signal pathway in the differentiation of BMSCs into enterocytes}

The above results in this study suggested that BMSCs could targeted migrate to intestinal injury sites and repair intestine damage as the result in improvements of endotoxemia and mortality. Thus, we assumed that BMSCs might be able to differentiate into enterocytes to repair intestinal epithelial barrier. According 
to previous reports, PI3K/AKT/mTOR signaling pathway is involved in cell fate and likely to be associated with proliferation and differentiation of stem cells [21, 22]. To investigate whether the PI3K/AKT/mTOR signaling pathway is associated with the differentiation of BMSCs into enterocytes, we performed the transwell co-culture system and evaluated the characteristic molecules of enterocytes by

immunofluorescence staining. CK and villin, two typical enterocytes molecules, revealed an abundance in BMSCs which were cultured with IEC-6 (Fig. 4a). Statistical analysis showed that after 10-day co-culture, the amounts of CK and villin in BMSCs with IEC- 6 were obviously more than those in BMSCs without IEC6 (Fig. 4b). Furthermore, the PI3K/AKT/mTOR signaling pathway inhibition resulted in significant decreases in CK and villin compared with the control groups (Fig. 4c and Fig. 4d). These data suggested that the PI3K/AKT/mTOR signaling pathway might be involved in the differentiation of BMSCs into enterocytes.

\section{Discussion}

In this study, we exhibited the characteristics of BMSCs from SD rats and showed the fine capacity for differentiation and proliferation, and high probability of PI3K/AKT/mTOR signaling pathway in inducing enterocytes differentiation. TAA-induced intestine injury based on acute liver failure was established appropriately and ameliorated by BMSC transplantation, which was revealed by a reduction in intestine damage, mortality, endotoxemia and inflammation.

The survival of patients with acute liver failure is closely related to the occurrence of hepatic coma, which usually caused by endotoxemia. Indeed, endotoxemia are observed in $75-93.3 \%$ of acute liver failure and severe hepatitis [23]. The common treatment such as reducing blood ammonia, artificial liver support system and transjugular intrahepatic portosystemic shunt (TIPS) can only relieve the symptoms, but cannot delay the progression of the delicate disease [24]. Up to now, with inspiring march in stem cell technology, an enthusiasm with cell-based therapy has been accumulated with the hope that the tragic outcome of liver failure can be saved. In recent years, with the welling up of studies on MSCs therapy for liver failure, solid evidences have consistently been showed that MSCs can effectively treat liver failure. In an open-label randomized controlled study, the administration of allogeneic BMSCs reduced serum total bilirubin (TBIL) and model for end-stage liver disease (MELD) scores in 56 patients with acute-on-chronic liver failure (ACLF) [25]. Some studies proved that MSCs could differentiate into hepatocyte-like cells (HLCs) in vivo, considered as one of the mechanisms of MSCs in the treatment of liver failure [26, 27]. While some experiments have suggested that MSCs cannot differentiate into HLCs after engrafting even if the improved survival, reduced alanine aminotransferase (ALT) and relieved liver injury [28]. These findings demonstrate that the effective performance of MSCs in the treatment of liver failure is not just dependent on the supplementing exogenous hepatocytes. The mechanism behind this therapeutic effect of MSCs may not have much to do with the liver. The similar effects between uninduced BMSCs and HLCs on the treatment of liver failure also confirms the above deduction from the side [29].

It is worth mentioning that some inflammatory factors including IL-6, TNF- $a$, and CD $8^{+} T$ cells, which are induced by bacteria and endotoxins (lipopolysaccharide, LPS) from gut, are significantly decreased after 
the BMSCs application in liver failure [30]. Previous studies have indicated that gut plays a significant role in endotoxemia based on liver failure. The level of plasma endotoxin in rats colectomized before administration of TAA had no difference from that in control group, the content of inflammatory factor and fatality were much lower [31]. We further investigated the role of BMSCs in gut and endotoxemia based on acute liver failure. The findings suggested that an appropriate dose of TAA could induce severe acute liver failure with damaged gut. After intragastric infusion of TAA, the BMSCs $\left(1 \times 10^{6}\right)$ were injected into caudal vein, the levels of plasma endotoxin and DAO were remarkably decreased compared to the TAA group. Also, excretion of IL- 6 and TNF-a decreased markedly, with the marked increase of survival rate. Histology with intestine from groups showed that the better intestinal mucosal barrier was observed after the BMSCs application. Moreover, the levels of IL- 6 and TNF-a in intestine were strongly reduced in rats with BMSCs. These findings suggested BMSCs were indeed effective in the treatment of liver failure and had important effects on intestinal injury and endotoxemia.

In this study, we used a fluorescence tracing approach to exhibit that BMSCs targeted migrated to intestinal injury sites. In order to confirm that BMSCs can differentiate into enterocytes and explore the possible pathway mechanism, we carried out the co-culture of BMSCs and IEC-6 in vitro using transwell culture medium. The data presented in this study with cocultivation medium, demonstrating the possibility of intestinal epithelioid differentiation of BMSCs with CK and villin overexpression, and the blocking of PI3K/AKT/mTOR signal pathway using LY294002 could interfere with this differentiation. $\mathrm{PI} 3 \mathrm{~K} / \mathrm{AKT} / \mathrm{mTOR}$ signal pathway has traditionally been considered a common mechanism of proliferation, differentiation and apoptosis. Previous studies have also mentioned the role of this pathway in stem cell differentiation, which is mutually verified with our research. Meanwhile, some previous studies have stated that the therapeutic effects of BMSCs are potentially based on its release of trophic and immunomodulatory factors such as transforming growth factor-beta (TGF- $\beta$ ) and interleukin 10 (IL-10) [32]. For example, BMSCs can inhibit cytotoxic CD8 ${ }^{+} \mathrm{T}$ lymphocyte (CTL) and NK cells through intercellular contact and paracrine factors including TGF- $\beta$ and IL-10, which have the immunosuppressive effects to alleviate inflammation. While endotoxemia and intestinal damage were strongly reduced in our model, we did not have a clear idea of the treatment mechanism behind this migration. In order to determine whether supplementing exogenous hepatocytes or protective cytokines secretion or even both, is the main treatment mechanism of BMSCs, further research is needed.

Of note, in recent studies about BMSCs therapy, the application of BMSCs could reduce the burden of liver failure in line with our results and attesting to the translational relevance, repair of intestinal mucosa reduced endotoxemia and mortality in a rat model of acute liver failure. Thus, it is beneficial to the treatment of acute liver failure and prevention from developing to endotoxemia using BMSCs. Clearing intestinal endotoxins and repairing intestinal damage should be paid more attention when against liver failure.

\section{Conclusions}


BMSCs from rats have a favorable impact on the application of stem cell-based therapy on TAA-induced acute liver failure. The two obvious advantages of this method lie in the repair of intestinal injury and the improvement of endotoxemia. Furthermore, the PI3K/AKT/mTOR signaling pathway is associated with the differentiation of BMSCs into enterocytes. These valuable data not only show us the role of intestinal endotoxemia, but also inspire us to build an effective therapy in the treatment of acute liver failure.

\section{Abbreviations}

MSCs: Mesenchymal stem cells; ESCs: Embryonic stem cells; iPSCs: Induced parthenogenetic stem cells; BMSCs: Bone marrow mesenchymal stem cells; PH: Partial hepatectomy; DMEM: Dulbecco's modified Eagle's medium; FBS: Fetal bovine serum; PBS: Phosphate buffer saline; PI: Propiony iodid; TAA:

Thioacetamide; H\&E: Hematoxylin and eosin; BrdU: Bromodeoxynucleoside uracil; DAO: Diamine oxidase; ELISA: Enzyme-linked immunosorbent assay; IL-6: Interleukin-6; TNF-a: Tumor necrosis factor- alpha; CK: Cytokeratin; IEC-6: Small intestinal crypt in rats; PI3K: Phosphoinositide kinase-3; AKT: Protein kinase B; mTOR: Mammalian target of rapamycin; SDS-PAGE: Sodium dodecyl sulfate-polyacrylamide gel electrophoresis; PVDF: Polyvinylidene fluoride; ANOVA: Analysis of variance; TIPS: Transjugular intrahepatic portosystemic shunt; TBIL: Total bilirubin; MELD: Model for end-stage liver disease; ACLF: Acute-on-chronic liver failure; HLCs: Hepatocyte-like cells; ALT: Alanine aminotransferase; LPS: Lipopolysaccharide; TGF- $\beta$ : Transforming growth factor-beta; IL-10: Interleukin 10; CTL: Cytotoxic T lymphocyte

\section{Declarations}

\section{Acknowledgements}

None

\section{Authors' contributions}

TJ, GX and BY conceived and coordinated the study, designed, performed, analyzed the experiments, and TJ wrote the paper. $\mathrm{HZ}$ and YY carried out data collection, data analysis, and revised the paper. CT and JY designed the study and revised the paper. All authors reviewed the results and approved the final version of the manuscript.

\section{Funding}

The work was financially supported by the National Natural Science Foundation of China (grant no. 81760107) and Scientific research fund project of Yunnan Provincial Department of Education (grant no. 2020Y0127).

\section{Availability of data and materials}


The datasets used and analyzed during the current study are available from the corresponding author on reasonable request.

\section{Ethics approval and consent to participate}

Animal care and all experimental procedures were performed in accordance with the protocol approved by Animal Experimental Ethical Committee of Kunming Medical University (Approval number KMMU2018020).

\section{Consent for publication}

Not applicable

\section{Competing interests}

The authors declare that they have no competing interests.

\section{References}

1. Stravitz RT, Lee WM. Acute liver failure. The Lancet. 2019;394(10201):869-81.

2. Thanapirom K, Treeprasertsuk S, Soonthornworasiri N, Poovorawan K, Chaiteerakij R, Komolmit P, et al. The incidence, etiologies, outcomes, and predictors of mortality of acute liver failure in Thailand: a population-base study. BMC Gastroenterology. 2019;19(1).

3. Wang Z, Wang A, Gong Z, Biviano I, Liu H, Hu J. Plasma claudin-3 is associated with tumor necrosis factor-alpha-induced intestinal endotoxemia in liver disease. Clinics Research in Hepatology Gastroenterology. 2019;43(4):410-6.

4. Mancini A, Campagna F, Amodio P, Tuohy KM. Gut: liver : brain axis: the microbial challenge in the hepatic encephalopathy. Food Funct. 2018;9(3):1373-88.

5. Weiler N, Schlotmann A, Schnitzbauer AA, Zeuzem S, Welker M-W. The epidemiology of acute liver failure. Deutsches Aerzteblatt Online. 2020.

6. Tan EX-X, Wang M-X, Pang J, Lee G-H. Plasma exchange in patients with acute and acute-on-chronic liver failure: A systematic review. World J Gastroenterol. 2020;26(2):219-45.

7. Wang XDARA, Andersson R, Soltesz V, Wang W, Svensson M, Bengmark $S$. The influence of surgically induced acute liver failure on the intestine in the rat. Scand J Gastroenterol. 1993;28(1):31-40.

8. Morgan J, Dibb M, Lal S. Intestinal failure-associated liver disease in adult patients. Current Opinion in Clinical Nutrition Metabolic Care. 2019;22(5):383-8.

9. Both SK, van Apeldoorn AA, Jukes JM, Englund MCO, Hyllner J, van Blitterswijk CA, et al. Differential bone-forming capacity of osteogenic cells from either embryonic stem cells or bone marrow-derived mesenchymal stem cells. Journal of Tissue Engineering Regenerative Medicine. 2011;5(3):180-90.

10. Wang L, Zhu M, Guo Q, Fan X, Lu Y, Zhu S, et al. Comparing the reprogramming efficiency of mouse embryonic fibroblasts, mouse bone marrow mesenchymal stem cells and bone marrow mononuclear 
cells to iPSCs. In Vitro Cellular \& Developmental Biology - Animal. 2012;48(4):236 - 43.

11. Danilova IG, Yushkov BG, Kazakova IA, Belousova AV, Minin AS, Abidov MT. Recruitment of macrophages and bone marrow stem cells to regenerating liver promoted by sodium phthalhydrazide in mice. Biomed Pharmacother. 2019;110:594-601.

12. Sun T, Li H, Bai Y, Bai M, Gao F, Yu J, et al. Ultrasound-targeted microbubble destruction optimized HGF-overexpressing bone marrow stem cells to repair fibrotic liver in rats. Stem Cell Research \& Therapy. 2020;11(1).

13. Wan Y-M, Li Z-q, Zhou Q, Liu C, Wang M-J, Wu H-X, et al. Mesenchymal stem cells alleviate liver injury induced by chronic-binge ethanol feeding in mice via release of TSG6 and suppression of STAT3 activation. Stem Cell Research \& Therapy. 2020;11(1).

14. Demirel U, Yalnız M, Aygün C, Orhan C, Tuzcu M, Sahin K, et al. Allopurinol Ameliorates Thioacetamide-Induced Acute Liver Failure by Regulating Cellular Redox-Sensitive Transcription Factors in Rats. Inflammation. 2012;35(4):1549-57.

15. Jiao F-Z, Wang Y, Zhang W-B, Zhang H-Y, Chen Q, Shi C-X, et al. Protective role of AGK2 on thioacetamide-induced acute liver failure in mice. Life Sci. 2019;230:68-75.

16. Milewski K, Hilgier W, Fręśko I, Polowy R, Podsiadłowska A, Zołocińska E, et al. Carnosine Reduces Oxidative Stress and Reverses Attenuation of Righting and Postural Reflexes in Rats with Thioacetamide-Induced Liver Failure. Neurochem Res. 2016;41(1-2):376-84.

17. Song H-Y, Sik Kim W, Kim J-M, Bak D-H, Moo Han J, Lim S-T, et al. A hydroxyethyl derivative of chrysin exhibits anti-inflammatory activity in dendritic cells and protective effects against dextran sodium salt-induced colitis in mice. Int Immunopharmacol. 2019;77:105958.

18. Yang NN, Ye Y, Tian ZX, Ma SM, Zheng Y, Huang J, et al. Effects of electroacupuncture on the intestinal motility and local inflammation are modulated by acupoint selection and stimulation frequency in postoperative ileus mice. Neurogastroenterology \& Motility. 2020.

19. Chen C, Liu Y-h, Cheng S-b, Wu S-I. Zhai X-j. The hepatoprotective effects of XCHD and MgIG against methotrexate-induced liver injury and inflammation in rats through suppressing the activation of AIM2 inflammasomes. Pathology - Research Practice. 2020;216(4):152875.

20. Han Y, Yu W, Zhang W, Yang Y, Walsh T, Oakeshott JG, et al. Variation in P450-mediated fenvalerate resistance levels is not correlated with CYP337B3 genotype in Chinese populations of Helicoverpa armigera. Pestic Biochem Physiol. 2015;121:129-35.

21. Chen Y, Fan Z, Wang X, Mo M, Zeng SB, Xu R-H, et al. PI3K/Akt signaling pathway is essential for de novo hair follicle regeneration. Stem Cell Research \& Therapy. 2020;11(1).

22. Qu Q, Pang Y, Zhang C, Liu L, Bi Y. Exosomes derived from human umbilical cord mesenchymal stem cells inhibit vein graft intimal hyperplasia and accelerate reendothelialization by enhancing endothelial function. Stem Cell Research \& Therapy. 2020;11(1).

23. DW H. Intestinal endotoxemia as a pathogenetic mechanism in liver failure. World J Gastroenterol. 2002;8(6):961-5. 
24. Amodio P. Hepatic encephalopathy: Diagnosis and management. Liver International. 2018;38(6):966-75.

25. Lin B-I, Chen J-f, Qiu W-h, Wang K-w, Xie D-y, Chen X-y, et al. Allogeneic bone marrow-derived mesenchymal stromal cells for hepatitis $B$ virus-related acute-on-chronic liver failure: A randomized controlled trial. Hepatology. 2017;66(1):209-19.

26. Yang J-F, Cao H-C, Pan Q-L, Yu J, Li J, Li L-J. Mesenchymal stem cells from the human umbilical cord ameliorate fulminant hepatic failure and increase survival in mice. Hepatobiliary Pancreatic Diseases International. 2015;14(2):186-93.

27. Liu S, Guo R, Hou X, Zhang Y, Jiang X, Wang T, et al. Adipose-tissue derived porcine mesenchymal stem cells efficiently ameliorate $\mathrm{CCl} 4$-induced acute liver failure in mice. Cytotechnology. 2020.

28. Chen G, Jin Y, Shi X, Qiu Y, Zhang Y, Cheng M, et al. Adipose-derived stem cell-based treatment for acute liver failure. Stem Cell Research \& Therapy. 2015;6(1).

29. Manzini BM, da Silva Santos Duarte A, Sankaramanivel S, Ramos AL, Latuf-Filho P, Escanhoela C, et al. Useful properties of undifferentiated mesenchymal stromal cells and adipose tissue as the source in liver-regenerative therapy studied in an animal model of severe acute fulminant hepatitis. Cytotherapy. 2015;17(8):1052-65.

30. Fang X, Liu L, Dong J, Zhang J, Song H, Song Y, et al. A study about immunomodulatory effect and efficacy and prognosis of human umbilical cord mesenchymal stem cells in patients with chronic hepatitis B-induced decompensated liver cirrhosis. J Gastroenterol Hepatol. 2018;33(4):774-80.

31. Liu LHD, Ren D. Effects of intestinal endotoxemia on pathogenesis of liver injury induced by thioacetamide. Zhonghua Gan Zang Bing Za Zhi. 2000;8(3):174-5.

32. Hu C-H, Tseng Y-W, Chiou C-Y, Lan K-C, Chou C-H, Tai C-S, et al. Bone marrow concentrate-induced mesenchymal stem cell conditioned medium facilitates wound healing and prevents hypertrophic scar formation in a rabbit ear model. Stem Cell Research \& Therapy. 2019;10(1).

\section{Tables}

Table 1 Histological criteria for the assessment of liver and intestine damage

\begin{tabular}{|c|c|c|}
\hline Score & Liver & Intestine \\
\hline 0 & no damage & no damage \\
\hline 1 & $\begin{array}{l}\text { minimal congestion and vacuolization, single-cell } \\
\text { necrosis }\end{array}$ & mild focal epithelial oedema and necrosis \\
\hline 2 & mild congestion and vacuolization, $\square 30 \%$ necrosis & moderate diffuse swelling and necrosis of the villi \\
\hline 3 & $\begin{array}{l}\text { moderate congestion and vacuolization, } 30 \%-60 \% \\
\text { necrosis }\end{array}$ & severe necrosis with evidence of neutrophil infiltration in the submucosa \\
\hline 4 & severe congestion and vacuolization, $\square 60 \%$ necrosis & $\begin{array}{l}\text { major widespread necrosis with massive neutrophil infiltration and evidence of } \\
\text { hemorrhage }\end{array}$ \\
\hline
\end{tabular}




\section{Figures}

\section{Figure 1}

Morphology of the BMSCs. (a) Passage 0 (P0) BMSCs at $\times 40$ magnification. (b) P3 BMSCs at $\times 40$ magnification. (c) and (d) The adipogenic differentiation of BMSCs and oil red $\mathrm{O}$ staining: control group (c) and adipogenic induction group of BMSCs (d), differentiation to adipocyte was demonstrated by Oil Red 0 staining, $\times 100$ magnification. (e) Cell cycle detection of BMSCs. (f) Most of BMSCs $(80.68 \%)$ were in $\mathrm{G} 1$ phase, which means that they have strong ability of cell proliferation. (g) FACS analysis of surface markers. (h) Typical hematologic lineage CD29 and CD90 were expressed in BMSCs, while CD 45 and CD CD34 were not.

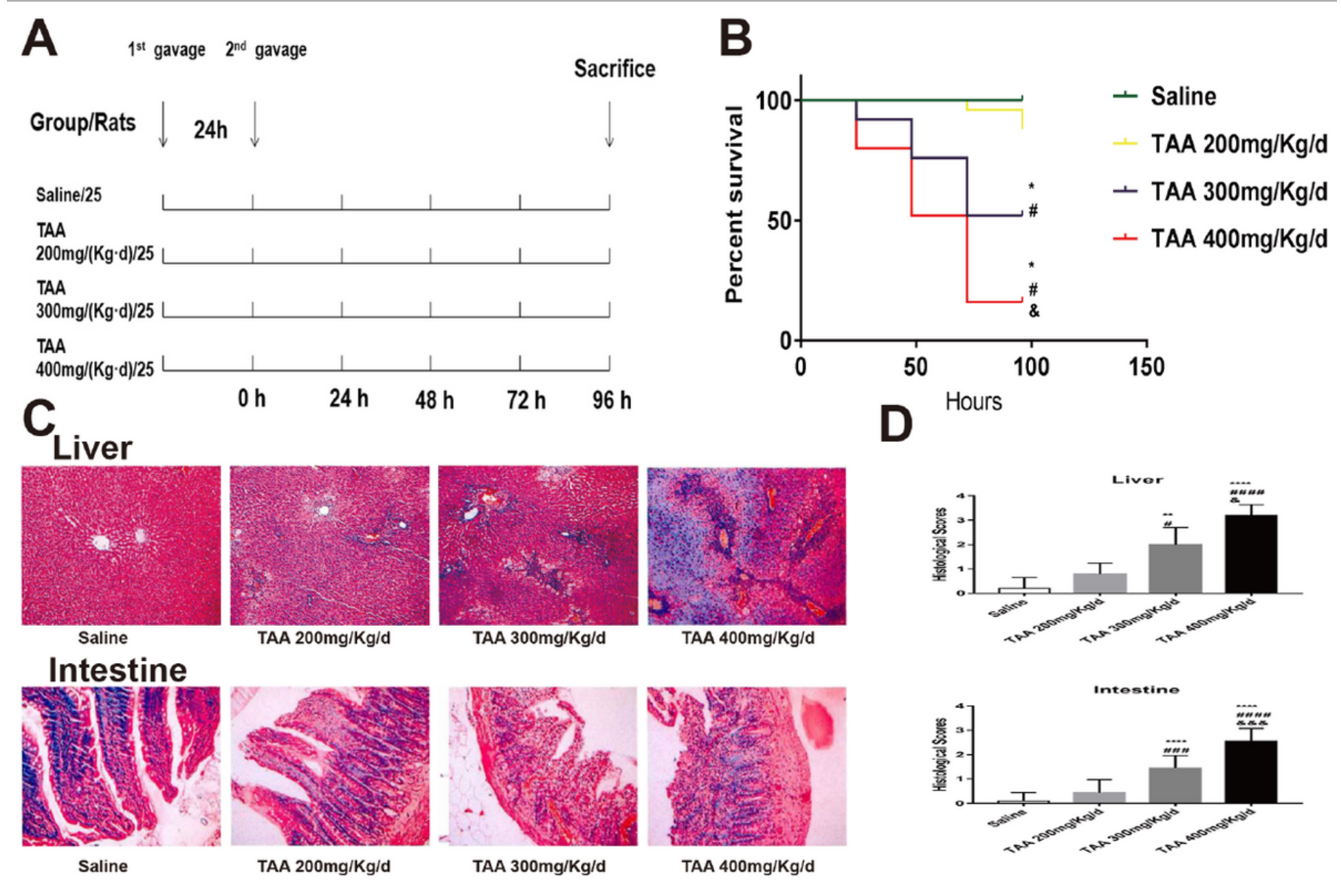

Figure 2

Animal model of acute liver failure induced by TAA. (a) Grouping and administration. (b) Survival analysis. ${ }^{*} p<0.05$ vs. saline; $\# p<0.05$ vs. TAA $200 \mathrm{mg} / \mathrm{Kg} / \mathrm{d}$; \& $p<0.05$ vs. TAA $300 \mathrm{mg} / \mathrm{Kg} / \mathrm{d}$. (c) H\&E staining, $\times 100$ magnification. (d) Histology analysis of four groups. ${ }^{\star \star} p<0.01,{ }^{\star \star \star \star} p<0.0001$ vs. saline; 
\# $p<0.05, \# \# \# p<0.001$, \#\#\#\# p < 0.0001vs. TAA 200mg/Kg/d; \& $p<0.05, \& \& \& p<0.001$ vs. TAA $300 \mathrm{mg} / \mathrm{Kg} / \mathrm{d}$.

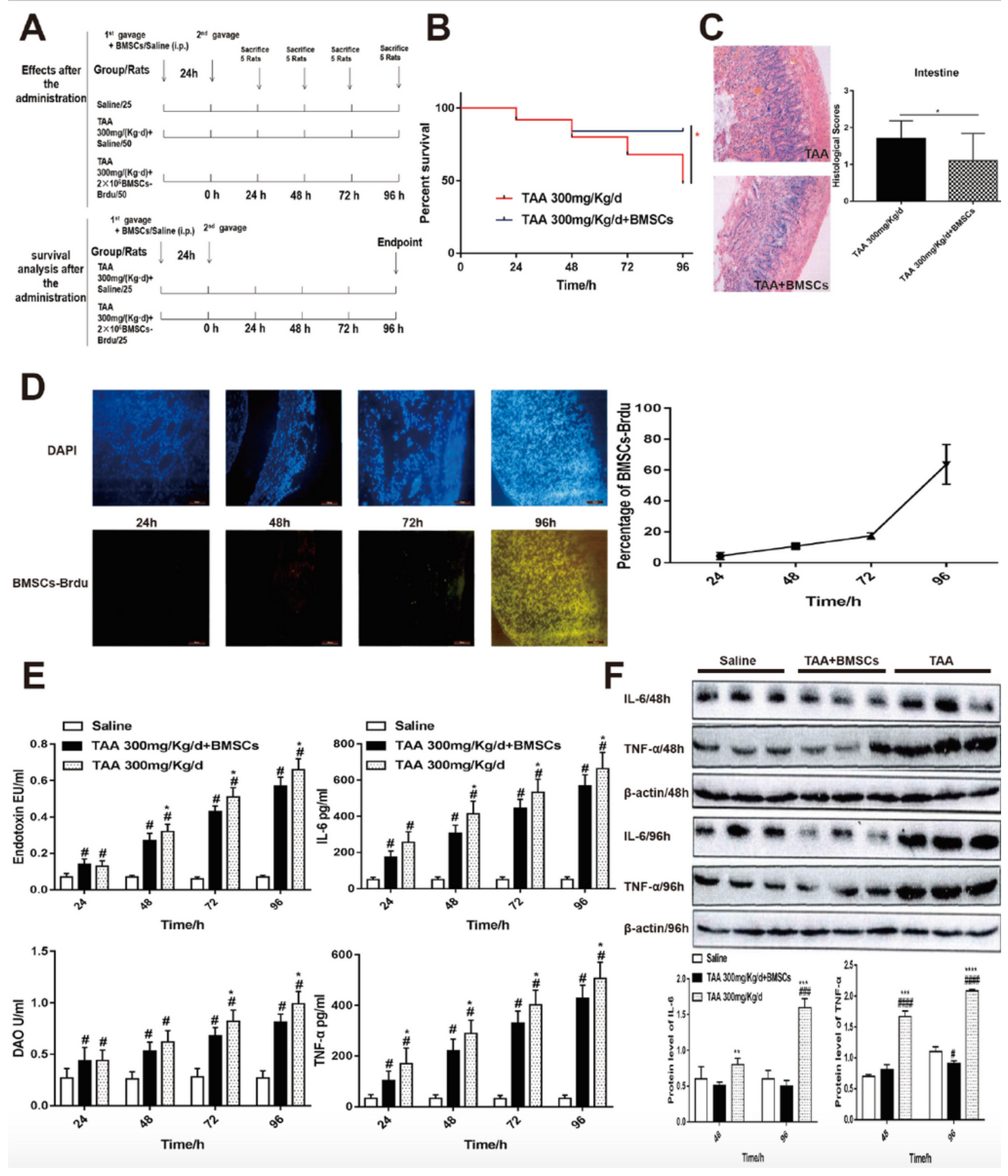

Figure 3

BMSCs administration alleviated acute liver failure in vivo. (a) Grouping and administration; Therapeutic experiment(upper) and mortality observation(lower). (b) Survival analysis. * $p<0.05$. (c) H\&E staining and histology analysis of intestines. * $p<0.05, \times 100$ magnification. (d) Tracing of BMSCs in rat 
intestines. DAPI-blue, BMSCs-Brdu-green. $\times 20$ magnification. (e) Differences of plasma endotoxin, DAO, IL- 6 and TNF- $a$ in rats at different time points after BMSCs administration. \# $p<0.05$ vs. the saline group at the same time. ${ }^{*} p<0.05$ vs. the TAA+BMSCs group at the same time. (f) Differences of intestinal IL- 6 and TNF- $a$ in rats at different time points after BMSCs administration. \#\#\# $p<0.001$, \#\#\#\#p<0.001vs. the saline group at the same time. ${ }^{* \star} p<0.01,{ }^{\star \star *} p<0.001,{ }^{\star \star \star *} p<0.0001$ vs. the TAA+BMSCs group at the same time.

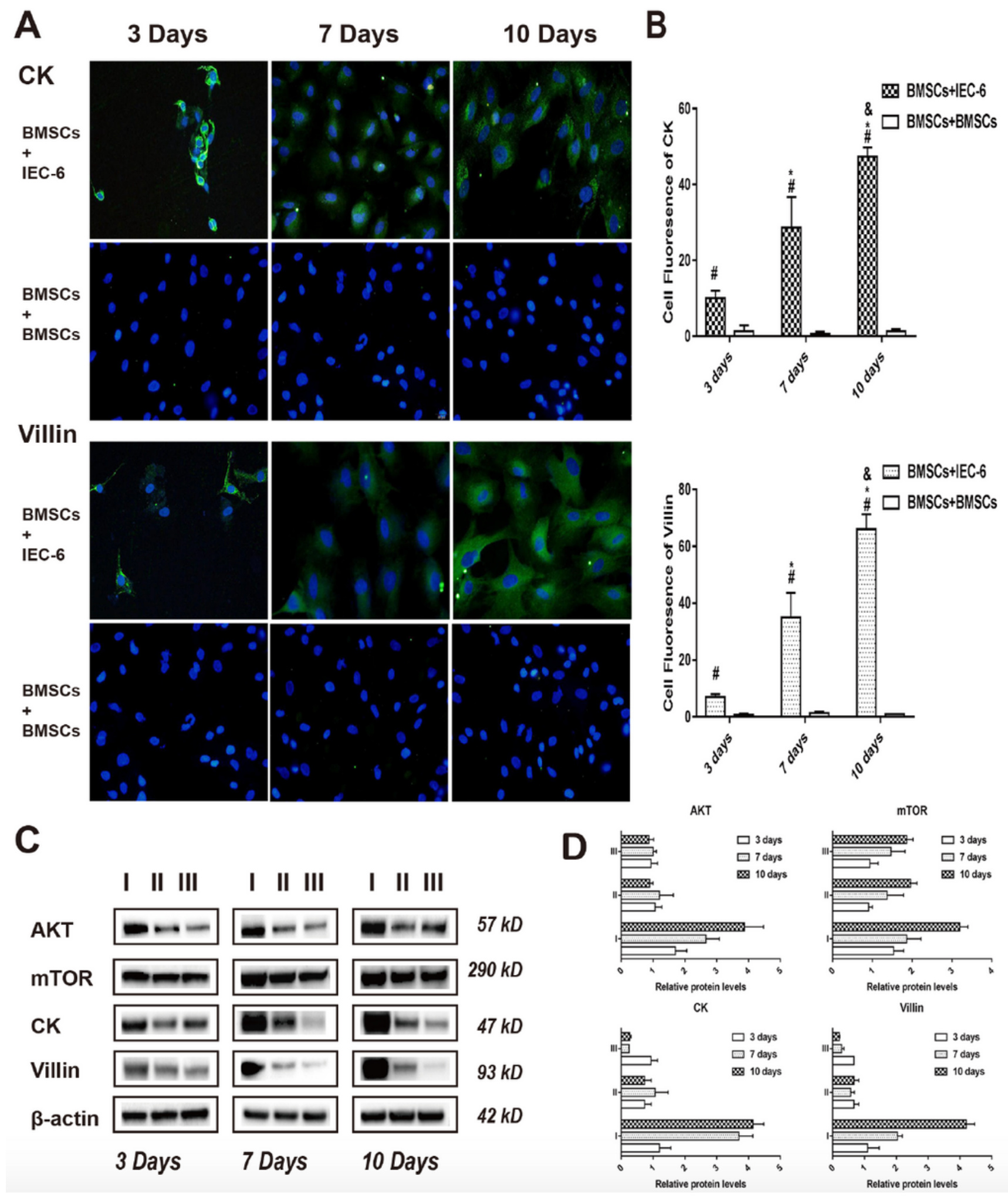

Figure 4 
$\mathrm{PI} 3 \mathrm{~K} / \mathrm{AKT} / \mathrm{mTOR}$ signaling pathway was involved in the differentiation of BMSCs into enterocytes in vitro. (a) Immunofluorescence staining was performed to exhibit CK and Villin expression (green staining) in the co-culture system at different 3 time points. DAPI-blue. $\times 40$ magnification. (b) Differences of CK and Villin in two co-culture systems. \# p<0.05, vs. the BMSCs + BMSCs group at the same time. ${ }^{*} p<$ 0.05 , vs. the BMSCs + IEC- 6 group at the 3 days. \& $p<0.05$, vs. the BMSCs + IEC- 6 group at the 7 days. (c) Western blot showed that blocking the PI3K/AKT/mTOR signal pathway interfered with the intestinal epithelial differentiation of BMSCs. Group I: IEC-6+BMSCs; Group II: IEC-6+LY294002+BMSCs; Group III: BMSCs+BMSCs. (d) Differences of AKT, mTOR, CK and Villin in 3 groups at different time points after PI3K blocker LY294002 administration. 